\title{
Orbital effect for the Fulde-Ferrell-Larkin-Ovchinnikov phase in a quasi-two-dimensional superconductor in a parallel magnetic field
}

\author{
A. G. Lebed* \\ Department of Physics, University of Arizona, 1118 E. 4-th Street, Tucson, Arizona 85721, USA
}

(Received 22 January 2018; revised manuscript received 16 March 2018; published 4 April 2018)

\begin{abstract}
We theoretically study the orbital destructive effect against superconductivity in a parallel magnetic field in the Fulde-Ferrell-Larkin-Ovchinnikov (FFLO or LOFF) phase at zero temperature in a quasi-two-dimensional (Q2D) conductor. We demonstrate that at zero temperature a special parameter, $\lambda=l_{\perp}(H) / d$, is responsible for strength of the orbital effect, where $l_{\perp}(H)$ is a typical "size" of the quasiclassical electron orbit in a magnetic field and $d$ is the interplane distance. We discuss applications of our results to the existing experiments on the FFLO phase in the organic Q2D conductors $\kappa-(\mathrm{ET})_{2} \mathrm{Cu}(\mathrm{NCS})_{2}$ and $\kappa-(\mathrm{ET})_{2} \mathrm{Cu}\left[\mathrm{N}(\mathrm{CN})_{2}\right] \mathrm{Cl}$.
\end{abstract}

DOI: 10.1103/PhysRevB.97.144504

It is well known that the orbital effect of electron motion in external magnetic field destroys superconductivity [1]. In singlet type-II superconductors, superconductivity is usually destroyed by magnetic fields higher than the so-called upper critical field, $H_{c 2}$. For a 3D isotropic case, at zero temperature $H_{c 2}(0)$ was calculated in Ref. [2], whereas temperature dependence of the upper critical field, $H_{c 2}(T)$, was found several years later [3]. As to triplet superconductivity, it can be restored in some cases in magnetic fields much higher than the $H_{c 2}(0)$, as theoretically predicted for quasi-one-dimensional (Q1D) [4,5], quasi-two-dimensional (Q2D) [6], and isotropic 3D [7] superconductors.

Note that superconductivity in singlet superconductors can also be destroyed by spin effects, as was first demonstrated in Refs. [8,9] (i.e., above the so-called Clogston-Chandrasekhar paramagnetic limit, $H_{P}$ ). Nevertheless, Larkin, Ovchinnikov, Felde, and Ferrell (LOFF) stressed [10,11] that the situation with the above mentioned paramagnetic destruction of superconductivity is not so simple. Indeed, they showed that there might exist the FFLO (or LOFF) superconducting nonuniform phase in the restricted area of magnetic fields, $H_{p}<H<$ $H_{\text {FFLO }}$. This happens when the orbital effect is small enough, which is realized in Q1D superconductors in an arbitrary oriented magnetic field and in Q2D superconductors for a magnetic field parallel to the conducting layers. As was shown in Ref. [12], the FFLO phase was stable in a pure 1D case for arbitrary strong magnetic field in the absence of the orbital effect. In Refs. [4,5,13,14], a possibility of the FFLO phase to exist in real Q1D materials from chemical family (TMTSF $)_{2} X$ ( $X=\mathrm{ClO}_{4}, \mathrm{PF}_{6}$, etc.) was studied taking into account the orbital effect in a perpendicular magnetic field. In Ref. [15], it was shown that the FFLO phase has to exist in the Q1D superconductor (TMTSF) ${ }_{2} \mathrm{ClO}_{4}$, despite the orbital effect in a parallel magnetic field. Some important signatures of the possible existence of the FFLO phase were experimentally observed

\footnotetext{
*Also at: L.D. Landau Institute for Theoretical Physics, RAS, 2 Kosygina Street, Moscow 117334, Russia.
}

in perpendicular $[16,17]$ and parallel $[18,19]$ magnetic fields in the Q1D superconductors (TMTSF) ${ }_{2} \mathrm{ClO}_{4}$ and (TMTSF) $)_{2} \mathrm{PF}_{6}$.

As mentioned before, the second convenient case for a possible observation of the FFLO phase is a Q2D superconductor in a parallel magnetic field. In the absence of the orbital effect (i.e., for a pure 2D case), such a problem was considered in Refs. [20-26] and some others (see for the references, reviews [23,24]). The orbital effect was first considered in Refs. [27,28] for high enough temperatures, $T \gg t_{\perp}$, for a Q2D superconductor with $t_{\perp} \ll T_{c 0}$. Here, $t_{\perp}$ is the overlapping integral of electron wave functions, corresponding to electron jumping in a perpendicular to the conducting planes direction, $T_{c 0}$ is superconducting transition temperature in the absence of a magnetic field. The main result of Refs. [27,28] is that the orbital effect is of the relative order of $t_{\perp}^{2} / T_{c 0}^{2} \ll 1$. From the experimental side, plenty of experimental works on Q2D organic and some other superconductors have been performed [29-41] to establish the possible existence of the FFLO phase in a parallel magnetic field.

The goal of our paper is to consider the orbital effect in a parallel magnetic field in a Q2D conductor at zero temperature, in contrast to Refs. [27,28]. We show that there exists a new parameter, $\lambda=l_{\perp}(H) / d$, where $l_{\perp}(H)$ is a typical "size" of the quasiclassical electron trajectory in a magnetic field and $d$ is the interlayer distance. We show that $\lambda$ defines how many conducting layers participate in the creation of one superconducting pair. In particular, we demonstrate that if this parameter is small, then we have effectively the superconducting pairing within almost one conducting layer and can disregard the orbital effect. On the contrary, if this parameter is large, then the superconducting pair is larger than the interlayer distance and it is necessary to take into account the orbital effect against superconductivity. We compare the obtained results with the existing experiments on the FFLO phase at low temperatures in $\kappa$-(ET) $)_{2} \mathrm{Cu}(\mathrm{NCS})_{2}$, where the FFLO phase has been the most firmly established [40].

Below, we consider a layered superconductor with the following Q2D electron spectrum:

$$
\epsilon(\mathbf{p})=\epsilon_{\|}\left(p_{x}, p_{y}\right)+2 t_{\perp} \cos \left(p_{z} d\right), \quad t_{\perp} \ll \epsilon_{F},
$$


in a parallel magnetic field,

$$
\mathbf{H}=(0, H, 0), \quad \mathbf{A}=(0,0,-H x),
$$

where $\hbar \equiv 1$. Here, in-plane electron energy $\epsilon_{\|}\left(p_{x}, p_{y}\right) \sim \epsilon_{F}$ with $\epsilon_{F}$ being the Fermi energy. Note that near 2D Fermi surface $(\mathrm{FS})$,

$$
\epsilon_{\|}\left(p_{x}, p_{y}\right)=\epsilon_{F},
$$

the Q2D electron spectrum (1) can be linearized:

$$
\epsilon(\mathbf{p})-\epsilon_{F}=v_{x}\left(p_{y}\right)\left[p_{x}-p_{x}\left(p_{y}\right)\right]+2 t_{\perp} \cos \left(p_{z} d\right),
$$

where $v_{x}\left(p_{y}\right)=\partial \epsilon_{\|}\left(p_{x}, p_{y}\right) / \partial p_{x}$ is a velocity component and $p_{x}\left(p_{y}\right)$ is the Fermi momentum component along the $x$ axis.

First, let us consider a qualitative physical picture of superconducting pairing in the magnetic field (2) and study the quasiclassical electron motion in the field. For simplicity, we employ an isotropic in-plane electron spectrum with

$$
\epsilon\left(p_{x}, p_{y}\right)=\frac{\left(p_{x}^{2}+p_{y}^{2}\right)}{2 m} .
$$

For electrons with spectrum (4),(5) the second Newton's law can be written in the magnetic field (2) as

$$
\frac{d p_{z}}{d t}=\left(\frac{e}{c}\right) v_{F} H \sin \alpha,
$$

where $\alpha$ is the angle between the magnetic field and electron position on the 2D FS (5). Note that electron velocity in perpendicular to the conducting planes direction can be written as $v_{z}\left(p_{y}\right)=2 t_{\perp} d \sin \left(p_{z} d\right)$ from Eq. (1). Therefore, electron oscillates in time in the perpendicular direction in the following way:

$$
z=z_{0}+\frac{2 t_{\perp} d}{\omega_{c} \sin \alpha} \sin \left(\omega_{c} \sin \alpha t\right), \omega_{c}=\frac{e v_{F} d H}{c},
$$

where

$$
l_{\perp}(H)=\frac{\lambda d}{\sin \alpha}, \lambda=\frac{4 t_{\perp}}{\omega_{c}}
$$

is a typical "size" of electron orbit in the magnetic field (2). From Eq. (8) it is directly seen that, at

$$
\lambda \ll 1,
$$

the most electrons are localized on conducting planes. This means that the orbital effect is small and, under this condition, we can expect that electrons form almost $2 \mathrm{D}$ superconducting pairs. Therefore, the FFLO phase is expected to survive.

Let us now consider the quantitative quantum problem of the FFLO phase formation in the presence of the orbital effect against superconductivity. To obtain electron Hamiltonian in the magnetic field (2), $\hat{H}\left(x ; p_{y}, p_{z}, s\right)$, we make use of the Peierls substitution method in Eq. (4) in the following way [6]:

$$
p_{x} \rightarrow-i\left(\frac{d}{d x}\right), p_{z} \rightarrow p_{z}+\left(\frac{e}{c}\right) H x,
$$

where $s= \pm \frac{1}{2}$ is electron spin projection along the quantization $y$ axis.

Under such conditions, the Green's functions of the Q2D electrons (4) in the magnetic field (2) obey the following differential equation [42],

$$
\begin{aligned}
{\left[i \omega_{n}-\hat{H}\left(x ; p_{y}, p_{z}, s\right)\right] G\left(i \omega_{n} ; x, x_{1} ; p_{y}, p_{z} ; s\right) } & =\delta\left(x-x_{1}\right), \\
\left\{i \omega_{n}-v_{x}\left(p_{y}\right)\left[-i \frac{d}{d x}-p_{x}\left(p_{y}\right)\right]+2 t_{\perp} \cos \left(p_{z} d+\frac{e H d x}{c}\right)+2 \mu_{B} H s\right\} G\left(i \omega_{n} ; x, x_{1} ; p_{y}, p_{z} ; s\right) & =\delta\left(x-x_{1}\right) .
\end{aligned}
$$

In Eq. (11), $\omega_{n}$ is the so-called Matsubara frequency [42] and $\mu_{B}$ is the Bohr magneton. Let us solve Eq. (11) analytically. As a result, for the Green's functions we obtain

$$
\begin{aligned}
G\left(i \omega_{n} ; x, x_{1} ; p_{y}, p_{z} ; s\right)= & -i \frac{\operatorname{sgn} \omega_{n}}{v_{x}\left(p_{y}\right)} \exp \left[-\frac{\omega_{n}\left(x-x_{1}\right)}{v_{x}\left(p_{y}\right)}\right] \times \exp \left\{\frac{i \lambda\left(p_{y}\right)}{2}\left[\sin \left(p_{z} d+\frac{e H d x}{c}\right)-\sin \left(p_{z} d+\frac{e H d x_{1}}{c}\right)\right]\right\} \\
& \times \exp \left[i p_{x}\left(p_{y}\right)\left(x-x_{1}\right)\right] \exp \left[\frac{2 i \mu_{B} s H\left(x-x_{1}\right)}{v_{x}\left(p_{y}\right)}\right],
\end{aligned}
$$

where $\lambda\left(p_{y}\right)=4 t_{\perp} c / e H d v_{x}\left(p_{y}\right)$.

To determine superconducting transition temperature as a function of a magnetic field, $T_{c}(H)$, we derive the so-called Gor'kov's equations [42] for the case of nonuniform superconductivity [4]. As a result, we obtain

$$
\begin{aligned}
\Delta(x)= & U \oint \frac{d l}{v_{\perp}(l)} \int_{\left|x-x_{1}\right|>\frac{\left|v_{x}(l)\right|}{\Omega}}^{\infty} \frac{2 \pi T d x_{1}}{v_{x}(l) \sinh \left[\frac{2 \pi T\left|x-x_{1}\right|}{v_{x}(l)}\right]} \\
& \times J_{0}\left\{2 \lambda(l) \sin \left[\frac{e H d\left(x-x_{1}\right)}{2 c}\right] \sin \left[\frac{e H d\left(x+x_{1}\right)}{2 c}\right]\right\} \times \cos \left[\frac{2 \mu_{B} H\left(x-x_{1}\right)}{v_{x}(l)}\right] \Delta\left(x_{1}\right),
\end{aligned}
$$

where integration in Eq. (13) is made along the $2 \mathrm{D}$ contour, $\epsilon_{\|}\left(p_{x}, p_{y}\right)=\epsilon_{F}, v_{\perp}(l)$ is a velocity component perpendicular to the contour, $U$ is an effective electron-electron interactions constant, $\Omega$ is a cutoff energy, and $J_{0}(\ldots)$ is the zero-order Bessel function. [Note that, for simplicity, Eq. (13) is derived for singlet $s$-wave superconductors].

We point out that Eq. (13) is the most general one among the existing equations to determine the parallel upper critical field in a layered s-wave superconductor. As the limiting cases, it contains Ginzburg-Landau and Lawrence-Doniach equations $[43,44]$ as well as quasiclassical equation similar to the gap equation of Ref. [2]. In particular, it takes into account quantum effects of 
electrons motion in a magnetic field—-the Bragg reflections—and related $3 D \rightarrow 2 D$ dimensional crossovers of electrons [6], which move in the extended Brillouin zone in a parallel magnetic field.

If we disregard the orbital effect in Eq. (13), it reduces to the following form

$$
1=U \oint \frac{d l}{v_{\perp}(l)} \int_{|z|>\frac{\left|v_{x}(l)\right|}{\Omega}}^{\infty} \frac{2 \pi T d z}{v_{x}(l) \sinh \left[\frac{2 \pi T|z|}{v_{x}(l)}\right]} \cos \left[\frac{2 \mu_{B} H z}{v_{x}(l)}\right] \cos \left[k_{1} z\right]
$$

defining the FFLO phase in a pure 2D case. Below, we consider the situation, where in the absence of the orbital effect small electron spectrum anisotropic effects fix the wave vector $k_{1}$ of the FFLO phase [28] and, thus, we have the following solution

$$
\Delta_{0}(x)=\cos \left(k_{1} x\right) .
$$

Here, we apply the in-plane magnetic field (2) perpendicular to the wave vector $k_{1}$ of the FFLO phase. Our task is to determine which fields can be considered as small ones and, thus, do not destroy the FFLO phase. We consider in-plane electron spectrum anisotropy to be large enough to fix the FFLO wave vector and to be small enough to influence the orbital effect [28]. In other words, in the presence of the orbital effects, we use the following equation, obtained for the isotropic in-plane electron spectrum (5):

$$
\begin{aligned}
\Delta(x)= & \frac{g}{2}\left\langle\int_{\left.\left|x-x_{1}\right|\right\rangle \frac{|\sin \alpha|}{\Omega}}^{\infty} \frac{2 \pi T d x_{1}}{v_{F} \sin \alpha \sinh \left[\frac{2 \pi T\left|x-x_{1}\right|}{v_{F} \sin \alpha}\right]}\right. \\
& \left.\times J_{0}\left\{\frac{2 \lambda}{\sin \alpha} \sin \left[\frac{e H d\left(x-x_{1}\right)}{2 c}\right] \sin \left[\frac{e H d\left(x+x_{1}\right)}{2 c}\right]\right\} \times \cos \left[\frac{2 \mu_{B} H\left(x-x_{1}\right)}{v_{F} \sin \alpha}\right] \Delta\left(x_{1}\right)\right\rangle_{\alpha},
\end{aligned}
$$

where $g$ is the effective electron coupling constant, $\langle\ldots\rangle_{\alpha}$ stands for averaging procedure over angle $\alpha$. Below, we introduce a more convenient variable, $z=(x-y) / \sin \alpha$. In this case, we can rewrite Eq. (16) in the following way:

$$
\Delta(x)=g \int_{\frac{v_{F}}{\Omega}}^{\infty} \frac{2 \pi T d z}{v_{F} \sinh \left(\frac{2 \pi T z}{v_{F}}\right)} \cos \left(\frac{2 \mu_{B} H z}{v_{F}}\right) \times\left\langle J_{0}\left\{\frac{2 \lambda}{\sin \alpha} \sin \left(\frac{\omega_{c} z \sin \alpha}{2 v_{F}}\right) \sin \left[\frac{\omega_{c}(2 x-z \sin \alpha)}{2 v_{F}}\right]\right\} \times \Delta(x-z \sin \alpha)\right\rangle_{\alpha} .
$$

Below, we treat the orbital effect against superconductivity as a small perturbation. To this end, we can expend the Bessel function in Eq. (17) with respect to a small parameter, $\lambda \ll 1,(8),(9)$ :

$$
\begin{aligned}
J_{0}\left\{\frac{2 \lambda}{\sin \alpha} \sin \left(\frac{z \omega_{c} \sin \alpha}{2 v_{F}}\right) \sin \left[\frac{\omega_{c}(2 x-z \sin \alpha)}{2 v_{F}}\right]\right\} \approx & -\frac{\lambda^{2}}{2 \sin ^{2} \alpha} \sin ^{2}\left(\frac{z \omega_{c} \sin \alpha}{2 v_{F}}\right) \\
& +\frac{\lambda^{2}}{2 \sin ^{2} \alpha} \cos \left(\frac{2 \omega_{c} x}{v_{F}}\right) \sin ^{2}\left(\frac{z \omega_{c} \sin \alpha}{2 v_{F}}\right) \cos \left(\frac{z \omega_{c} \sin \alpha}{v_{F}}\right) \\
& +\frac{\lambda^{2}}{2 \sin ^{2} \alpha} \sin \left(\frac{2 \omega_{c} x}{v_{F}}\right) \sin ^{2}\left(\frac{z \omega_{c} \sin \alpha}{2 v_{F}}\right) \sin \left(\frac{z \omega_{c} \sin \alpha}{v_{F}}\right) .
\end{aligned}
$$

It is possible to make sure that Eqs. (17) under the approximation (18) has the following solution at $T=0$ :

$$
\Delta(x)=\cos \left(k_{1} x\right)+A \cos \left(k_{1} x\right) \cos \left(k_{2} x\right)+B \sin \left(k_{1} x\right) \sin \left(k_{2} x\right),
$$

where $k_{1}=2 \mu_{B} / v_{F}$ and $k_{2}=2 \omega_{c} / v_{F} ; A \sim B \sim \lambda^{2}$. Note that, in Eqs. (18) and (19), we keep only terms of the order of $\lambda^{2}$ and disregard all terms of the order of $\lambda^{4}$ or less. After substituting Eqs. (18) and (19) into integral Eq. (17) and disregarding all terms of the order of $\lambda^{4}$, we obtain the following three equations at $T=0$ :

$$
\begin{gathered}
\frac{1}{g}=\int_{\frac{v_{F}}{\Omega}}^{\infty} \frac{d z}{z}\left\langle\left[1-\frac{\lambda^{2}}{2 \sin ^{2} \alpha} \sin ^{2}\left(\frac{k_{2} z \sin \alpha}{4}\right)\right] \times \cos \left(k_{1} z\right) \cos \left(k_{1} z \sin \alpha\right)\right\rangle_{\alpha}, \\
(A+B)\left\{\frac{1}{g}-\int_{\frac{v_{F}}{\Omega}}^{\infty} \frac{d z}{z} \cos \left(k_{1} z\right) J_{0}\left[\left(k_{1}-k_{2}\right) z\right]\right\}=\frac{\lambda^{2}}{2} \int_{\frac{v_{F}}{\Omega}}^{\infty} \frac{d z}{z}\left\langle\left.\frac{1}{\sin ^{2} \alpha} \sin ^{2}\left(\frac{k_{2} z \sin \alpha}{4}\right) \cos \left[\frac{\left(k_{1}-k_{2}\right) z \sin \alpha}{2}\right] \cos \left(k_{1} z\right)\right|_{\alpha}\right. \\
(A-B)\left\{\frac{1}{g}-\int_{\frac{v_{F}}{\Omega}}^{\infty} \frac{d z}{z} \cos \left(k_{1} z\right) J_{0}\left[\left(k_{1}+k_{2}\right) z\right]\right\}=\frac{\lambda^{2}}{2} \int_{\frac{v_{F}}{\Omega}}^{\infty} \frac{d z}{z}\left\langle\frac{1}{\sin ^{2} \alpha} \sin ^{2}\left(\frac{k_{2} z \sin \alpha}{4}\right) \cos \left[\frac{\left(k_{1}+k_{2}\right) z \sin \alpha}{2}\right] \cos \left(k_{1} z\right)\right\rangle_{\alpha}
\end{gathered}
$$

Note that Eq. (20) defines correction to the FFLO critical magnetic field due to the orbital effect at $T=0$, whereas Eqs. (21) and (22) define corrections (19) to the FFLO solution (15).

In this paper, we restrict our analysis by calculation of correction (20) to the FFLO critical magnetic field, $H_{\mathrm{FFLO}}$. Let us recall that, in the absence of a magnetic field, Eq. (17) reduces to:

$$
\frac{1}{g}=\int_{\frac{v_{F}}{\Omega}}^{\infty} \frac{2 \pi T_{c 0} d z}{v_{F} \sinh \left(\frac{2 \pi T_{c 0} z}{v_{F}}\right)}
$$


where $T_{c 0}$ is the superconducting transition temperature at $H=$ 0 . Note that, if orbital effect is negligible (i.e., at $\lambda=0$ ), then the FFLO critical magnetic field, $H_{\text {FFLO }}$ satisfy the following equation (20):

$$
\int_{\frac{v_{F}}{\Omega}}^{\infty} \frac{2 \pi T_{c 0} d z}{v_{F} \sinh \left(\frac{2 \pi T_{c 0} z}{v_{F}}\right)}=\int_{\frac{v_{F}}{\Omega}}^{\infty} \frac{d z}{z} \cos \left(k_{1} z\right) J_{0}\left(k_{1} z\right)
$$

where

$$
J_{0}\left(k_{1} z\right)=\left\langle\cos \left(k_{1} z \sin \alpha\right)\right\rangle_{\alpha}
$$

As shown in Ref. [20], in a pure 2D case, Eq. (24) has the following solution (see also Ref. [45]):

$$
H_{\mathrm{FFLO}}=\frac{\Delta_{0}}{\mu_{B}}=\frac{\pi k_{b} T_{c 0}}{2 \gamma}
$$

where $\Delta_{0}$ is a superconducting gap in the Bardeen-CooperSchrieffer theory [1], $k_{B}$ is the Boltzmann constant, and $\gamma$ is the Euler constant [45].

Therefore, Eq. (20) can be rewritten as

$$
\ln \left(H_{\mathrm{FFLO}} / H_{\mathrm{FFLO}}^{*}\right)=\lambda^{2} \int_{0}^{\infty} \frac{d z}{z} \times\left\langle\frac{\sin ^{2}\left(k_{2} z \sin \alpha / 4\right)}{2 \sin ^{2} \alpha} \cos \left(k_{1} z\right) \cos \left(k_{1} z \sin \alpha\right)\right\rangle_{\alpha},
$$

where $H_{\mathrm{FFLO}}^{*}$ is the critical field of the FFLO phase in the presence of the orbital effect. In this paper we consider the case of the small orbital effect (9), thus, Eq. (27) can be rewritten in the following way:

$$
\left(H_{\mathrm{FFLO}}-H_{\mathrm{FFLO}}^{*}\right) / H_{\mathrm{FFLO}}=\lambda^{2} \int_{0}^{\infty} \frac{d z}{z} \times\left\langle\frac{\sin ^{2}\left(k_{2} z \sin \alpha / 4\right)}{2 \sin ^{2} \alpha} \cos \left(k_{1} z\right) \cos \left(k_{1} z \sin \alpha\right)\right\rangle_{\alpha} .
$$

It is possible to make sure that the integral in Eqs. (27) and (28) is a convergent one. Moreover, the integral is small since it is proportional to $\lambda^{2} \ll 1$ and, thus, the FFLO phase is stable even in the presence of the orbital effect. As we have already discussed in the "qualitative" part of this paper, physically this means that the FFLO superconducting pair is located mostly within one conducting layer. Under such a condition (9), the intralayer currents are small and, in fact, we have coexistence of the FFLO phase $[10,11]$ and the reentrant superconductivity [4-6].

Let us demonstrate that the above mentioned situation corresponds to the existence of the FFLO phase in the Q2D superconductor $\kappa-(\mathrm{ET})_{2} \mathrm{Cu}(\mathrm{NCS})_{2}$, where, in our opinion, it is the most firmly experimentally established [40]. Indeed, if we take experimental value of the perpendicular upper critical field, $H_{c 2}^{\perp} \simeq 5 T$, we obtain the Ginzburg-Landau parallel coherence length $\xi_{\|} \simeq 0.8 \times 10^{-6} \mathrm{~cm}$ from the standard equation: $H_{c 2}^{\perp}=$ $\tau \phi_{0} /\left(2 \pi \xi_{\|}^{2}\right)$, where $\tau=\left(T_{c 0}-T\right) / T_{c 0}, \phi_{0}$ is the flux quantum. Then, from the equation $\xi_{\|}=\sqrt{7 \zeta(3)} v_{F} /\left(4 \sqrt{2} \pi T_{c 0}\right)$ [46], we find the value of in-plane Fermi velocity, $v_{F} \simeq 0.65 \times$ $10^{7} \mathrm{~cm} / \mathrm{s}$. If we take into account that the interplane distance is $d=1.62 \times 10^{-7} \mathrm{~cm}$, we obtain the cyclotron frequency (7): $\omega_{c}(H) / H \simeq 1.23 \mathrm{~K} / \mathrm{T}$. So, in the integral (28), $k_{2} / k_{1} \simeq 1.85$ and, as it is possible to show, its numerical evaluation gives the value of 0.12 . Therefore, Eq. (28) can be rewritten as

$$
H_{\mathrm{FFLO}}-H_{\mathrm{FFLO}}^{*}=0.12 \lambda^{2} \text {. }
$$

Estimation of $t_{\perp} \simeq 2 \mathrm{~K}$ [47], gives us the following value of parameter $\lambda=0.16$ in the vicinity of the magnetic field $H_{\mathrm{FFLO}} \simeq 27.5 \mathrm{~T}$. So, we can conclude that indeed, in the Q2D superconductor $\kappa$-(ET) $)_{2} \mathrm{Cu}(\mathrm{NCS})_{2}$, the FFLO phase coexists with the reentrant superconductivity. Note that qualitatively the above mentioned statement does not depend on actual symmetry of superconducting gap, which may be $d$ wave in the $\kappa-(\mathrm{ET})_{2} \mathrm{Cu}(\mathrm{NCS})_{2}$.

Let us consider another relative $\mathrm{Q} 2 \mathrm{D}$ organic conductor$\kappa-(\mathrm{ET})_{2} \mathrm{Cu}\left[\mathrm{N}(\mathrm{CN})_{2}\right] \mathrm{Cl}$ (see, for example, Ref. [31]). In accordance with [31], in this case under pressure $P=1.9 \mathrm{kbar}$,
$T_{c 0} \simeq 7 \mathrm{~K}$, and $H_{c 2}^{\perp} \simeq 2 \mathrm{~T}$. Using the same equations as before, we obtain $\xi_{\|} \simeq 1.25 \times 10^{-6} \mathrm{~cm}$ and $v_{F} \simeq 0.73 \times 10^{7} \mathrm{~cm} / \mathrm{s}$. Moreover, from Ref. [31], it follows that $H_{c 2}^{\|} \simeq 20 \mathrm{~T}$ and, using equation $\xi_{\perp}=\sqrt{7 \zeta(3)} 2 t_{\perp} d /\left(4 \sqrt{2} \pi T_{c 0}\right)$ [46], we obtain $t_{\perp} \simeq 17 \mathrm{~K}$. Taking into account that $d \simeq 1.5 \times 10^{-7} \mathrm{~cm}$, we find that, in this case, the parameter $\lambda \simeq 2.6$ is large and Eq. (18) is not valid. In other words, the orbital effect against superconductivity is important and, thus, it is necessary to solve Eq. (17) directly for $\lambda \geqslant 1$. However, this is beyond the scope of the current paper.

To summarize, we have shown that, at small values of the parameter $\lambda \ll 1$ in Eq. (8), the superconducting FFLO phase in a parallel magnetic field occupies almost one conducting layer at $T=0$. In this case, the FFLO phase $[10,11]$ exists under the reentrant superconductivity regime [4-6] and the correction from the orbital effect to the FFLO critical magnetic field (28) is small. Such a situation has been shown to exist in the Q2D superconductor $\kappa-(\mathrm{ET})_{2} \mathrm{Cu}(\mathrm{NCS})_{2}$. If parameter $\lambda$ is of the order of unity, as it is in the case of another Q2D organic conductors $\kappa-(\mathrm{ET})_{2} \mathrm{Cu}\left[\mathrm{N}(\mathrm{CN})_{2}\right] \mathrm{Cl}$, then the orbital effect becomes large and Eq. (17) needs to be solved without expanding the Bessel function. The latter problem is very difficult from a numerical point of view and hopefully will be considered in the future. We stress that our results are different from that in Refs. [27,28], since at zero temperature it is not possible to expand the superconducting gap equation with respect to parameter $t_{\perp} / T$. In the end of the paper, we discuss in a brief one delicate property of our model - that the direction of the FFLO phase is supposed to be unchanged in a magnetic field. This definitely works for the case of small magnetic fields, considered in the paper, where anisotropy of the 2D FS fixes the FFLO direction. As to relatively high magnetic fields, the effect of changing of the FFLO direction has to be somehow added to Eq. (17).

We are thankful to C.C. Agosta, N.N. Bagmet (Lebed), and M.V. Kartsovnik for useful discussions. 
[1] See, for example, A. A. Abrikosov, Fundamentals of Theory of Metals (Elsevier Science, Amsterdam, 1988).

[2] L. P. Gor'kov, Sov. Phys. JETP 37, 42 (1960).

[3] N. R. Werthamer, E. Helfand, and P. C. Hohenberg, Phys. Rev. 147, 295 (1966).

[4] A. G. Lebed, JETP Lett. 44, 114 (1986) [Pis'ma Zh. Eksp. Teor. Fiz. 44, 89 (1986)].

[5] N. Dupuis, G. Montambaux, and C. A. R. Sa de Melo, Phys. Rev. Lett. 70, 2613 (1993).

[6] A. G. Lebed and K. Yamaji, Phys. Rev. Lett. 80, 2697 (1998).

[7] M. Razolt and Z. Tesanovic, Rev. Mod. Phys. 64, 709 (1992).

[8] A. M. Clogston, Phys. Rev. Lett. 9, 266 (1962).

[9] B. S. Chandrasekhar, Appl. Phys. Lett. 1, 7 (1962).

[10] A. I. Larkin and Yu. N. Ovchinnikov, Zh. Eksp. Teor. Fiz. 47, 1136 (1964) [Sov. Phys. JETP 20, 762 (1965)].

[11] P. Fulde and R. A. Ferrell, Phys. Rev. 135, A550 (1964).

[12] A. I. Buzdin and V. V. Tugushev, Zh. Eksp. Teor. Phys. 85, 735 (1983) [Sov. Phys. JETP. 58, 428 (1983)].

[13] N. Dupuis, Phys. Rev. B 51, 9074 (1995).

[14] A. G. Lebed, Phys. Rev. Lett. 107, 087004 (2011); Phys. Rev. B 59, R721 (1999).

[15] A. G. Lebed and S. Wu, Phys. Rev. B 82, 172504 (2010).

[16] I. J. Lee, M. J. Naughton, G. M. Danner, and P. M. Chaikin, Phys. Rev. Lett. 78, 3555 (1997).

[17] J. I. Oh and M. J. Naughton, Phys. Rev. Lett. 92, 067001 (2004).

[18] S. Yonezawa, S. Kusaba, Y. Maeno, P. Auban-Senzier, C. Pasquier, K. Bechgaard, and D. Jerome, Phys. Rev. Lett. 100, 117002 (2008).

[19] S. Yonezawa, S. Kusaba, Y. Maeno, P. Auban-Senzier, C. Pasquier, and D. Jerome, J. Phys.: Conf. Ser. 150, 052289 (2009).

[20] L. N. Bulaevskii, Zh. Eksp. Teor. Fiz. 65, 1278 (1973) [Sov. Phys. JETP 38, 634 (1974)].

[21] H. Shimahara, Phys. Rev. B 50, 12760 (1994).

[22] H. Shimahara, J. Phys. Soc. Jpn. 67, 736 (1998).

[23] See chapter by H. Shimahara in The Physics of Organic Superconductors and Conductors, edited by A. G. Lebed (Springer, Berlin, 2008).

[24] A. I. Buzdin, Rev. Mod. Phys. 77, 935 (2005).

[25] A. I. Buzdin and J. P. Brison, Phys. Lett. A 218, 359 (1996).

[26] A. I. Buzdin and J. P. Brison, Europhys. Lett 35, 707 (1996).

[27] M. D. Croitoru and A. I. Buzdin, Phys. Rev. B 86, 064507 (2012).

[28] M. D. Croitoru, M. Houzet, and A. I. Buzdin, Phys. Rev. Lett. 108, 207005 (2012).
[29] J. Singleton, J. A. Symington, M.-S. Nam, A. Ardavan, M. Kurmoo, and P. Day, J. Phys.: Condens. Matter 12, L641 (2000).

[30] M. A. Tanatar, T. Ishiguro, H. Tanaka, and H. Kobayashi, Phys. Rev. B 66, 134503 (2002).

[31] Y. Shimojo, T. Ishiguro, H. Yamoji, and G. Saito, J. Phys. Soc. Jpn. 71, 1716 (2002).

[32] R. Lortz, Y. Wang, A. Demuer, P. H. M. Böttger, B. Bergk, G. Zwicknagl, Y. Nakazawa, and J. Wosnitza, Phys. Rev. Lett. 99, 187002 (2007).

[33] K. Cho, B. E. Smith, W. A. Coniglio, L. E. Winter, C. C. Agosta, and J. A. Schlueter, Phys. Rev. B 79, 220507 (2009).

[34] B. Bergk, A. Demuer, I. Sheikin, Y. Wang, J. Wosnitza, Y. Nakazawa, and R. Lortz, Phys. Rev. B 83, 064506 (2011).

[35] W. A. Coniglio, L. E. Winter, K. Cho, C. C. Agosta, B. Fravel, and L. K. Montgomery, Phys. Rev. B 83, 224507 (2011).

[36] J. A. Wright, E. Green, P. Kuhns, A. Reyes, J. Brooks, J. Schlueter, R. Kato, H. Yamamoto, M. Kobayashi, and S. E. Brown, Phys. Rev. Lett. 107, 087002 (2011).

[37] C. C. Agosta, J. Jin, W. A. Coniglio, B. E. Smith, K. Chao, I. Stroe, C. Martin, S. W. Tozer, T. P. Murphy, E. C. Palm et al., Phys. Rev. B 85, 214514 (2012).

[38] H. Mayaffre, S. Kramer, M. Horvatic, C. Berthier, K. Miyagawa, K. Kanoda, and V. F. Mitrovic, Nat. Phys. 10, 928 (2014).

[39] S. Tsuchiya, J.-I. Yamada, K. Sugii, D. Graf, J. S. Brooks, T. Terashima, and S. Uji, J. Phys. Soc. Jpn. 84, 034703 (2015).

[40] C. C. Agosta, N. A. Fortune, Scott T. Hannahs, S. Gu, L. Liang, J.-H. Park, and J. A. Schleuter, Phys. Rev. Lett. 118, 267001 (2017).

[41] C.-W. Cho, J. H. Yang, N. F. Q. Yuan, J. Shen, T. Wolf, and R. Lortz, Phys. Rev. Lett. 119, 217002 (2017).

[42] A. A. Abrikosov, L. P. Gor'kov, and I. E. Dzyaloshinskii, Methods of Quantum Field Theory in Statistical Mechanics (Dover, New York, 1963).

[43] L. N. Bulaevskii and A. A. Guseinov, Pis'ma Zh. Eksp. Teor. Fiz. 19, 742 (1974) [JETP Lett. 19, 382 (1974)].

[44] R. A. Klemm, A. Luther, and M. R. Beasley, Phys. Rev. B 12, 877 (1975).

[45] I. S. Gradshteyn and I. M. Ryzhik, Table of Integrals, Series, and Products, 6th ed. (Academic Press, London, 2000).

[46] L. P. Gor'kov and T. K. Melik-Barkhudarov, ZhETF 45, 1493 (1964) [Sov. Phys. JETP 18, 1031 (1964)].

[47] P. A. Goddard, S. J. Blundell, J. Singleton, R. D. McDonald, A. Ardavan, A. Narduzzo, J. A. Schlueter, A. M. Kini, and T. Sasaki, Phys. Rev. B 69, 174509 (2004). 